\title{
A randomised phase II trial of selumetinib vs selumetinib plus temsirolimus for soft-tissue sarcomas
}

\author{
Z Eroglu ${ }^{1}, \mathrm{H}$ A Tawbi ${ }^{2}, \mathrm{~J} \mathrm{Hu}^{3}, \mathrm{M} \mathrm{Guan}{ }^{1}, \mathrm{P} \mathrm{H} \mathrm{Frankel}^{1}, \mathrm{~N} \mathrm{H}$ Ruel $^{1}$, S Wilczynski ${ }^{1}$, S Christensen ${ }^{4}$, D R Gandara ${ }^{4}$ \\ and W A Chow ${ }^{\star}, 1$ \\ ${ }^{1}$ City of Hope National Medical Center, Duarte, CA, USA; ${ }^{2}$ University of Pittsburgh Cancer Institute and University of Pittsburgh \\ School of Medicine, Pittsburgh, PA, USA; ${ }^{3}$ Norris Comprehensive Cancer Center, University of Southern California, Los Angeles, \\ CA, USA and ${ }^{4}$ University of California Davis Comprehensive Cancer Center, Sacramento, CA, USA
}

Background: The MEK inhibitor, selumetinib, suppresses soft-tissue sarcoma (STS) cell proliferation in vitro. Mammalian target of rapamycin inhibitors possess modest activity against STS; however, resistance develops via MAPK pathway feedback activation. The combination of selumetinib and temsirolimus synergistically inhibits STS cell line growth. Therefore, a randomized phase II trial of selumetinib vs selumetinib plus temsirolimus was conducted.

Methods: Seventy-one adults with advanced STS who received $\leqslant 2$ prior chemotherapeutics were randomized to selumetinib $75 \mathrm{mg}$ p.o. bid and allowed to crossover upon progression, or to selumetinib $50 \mathrm{mg}$ p.o. bid plus temsirolimus $20 \mathrm{mg}$ i.v. weekly, with primary endpoint of progression-free survival (PFS).

Results: There was no difference in PFS between the two arms for the overall cohort (median 1.9 vs 2.1 months); an improved median PFS was observed in the combination arm $(N=11)$ over single agent $(N=10)$ in the prespecified leiomyosarcoma stratum (median 3.7 vs 1.8 months; $P=0.01$ ). Four-month PFS rate was 50\% (95\% confidence interval $0.19-0.81$ ) with the combination vs $0 \%$ with selumetinib alone in the leiomyosarcoma cohort. Most common grade 3/4 adverse events with the combination were mucositis (29\%), lymphopenia (26\%), neutropenia and anaemia (20\% each).

Conclusions: While single-agent selumetinib has no significant activity in STS, the combination may be active for leiomyosarcomas.

Soft-tissue sarcomas (STS) are a heterogeneous group of malignant, mesenchymal tumours that are comprised of over 50 different subtypes. Outcomes for recurrent STS are often poor because of their relative resistance to chemotherapy. Chemotherapy with doxorubicin alone or in combination with ifosfamide is often used in the first-line setting and the combination of gemcitabine and docetaxel is commonly used in the second-line setting. Pazopanib, a vascular endothelial growth factor receptor inhibitor, was the first targeted therapy agent to be approved in STS. The FDA approval is based upon a phase 3 trial in the second- or third-line setting and showed a median progression-free survival (PFS) of 4.6 vs 1.6 months with placebo.(van der Graaf et al, 2012) Cotargeting signalling pathways may be a promising strategy to overcome resistance pathways that rapidly develop during therapy for STS.

The mammalian target of rapamycin (mTOR) is a protein kinase that regulates protein translation, cell growth, autophagy, and apoptosis.(Sabatini, 2006) Intracellular signalling through mTOR and associated upstream signalling pathways are dysregulated in most sarcoma subtypes. (Wan and Helman, 2007)

*Correspondence: DrWA Chow; E-mail: wchow@coh.org

Presented at the 50th Annual Meeting of the American Society of Clinical Oncology, Chicago, IL, May 30-June 3, 2014

Received 3 December 2014; revised 24 February 2015; accepted 9 March 2015;

published online 21 April 2015

(C) 2015 Cancer Research UK. All rights reserved 0007-0920/15

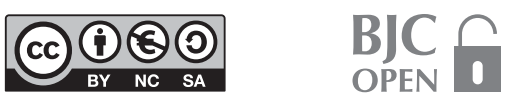


Mammalian target of rapamycin is regulated in part by AKT, a serine/threonine kinase. Mammalian target of rapamycin inhibitors (mTORi) may increase PI3K activity towards MAPK activation in a feedback activation loop, therefore, promoting both AKT and ERK phosphorylation.(Kinkade et al, 2008) (Figure 2A) The insulin-like growth factor 1 receptor (IGF-1R) pathway is a commonly activated pathway in a variety of sarcomas, and the MAPK pathway is also downstream of IGF-1R (Wan and Helman, 2007). In preclinical breast and prostate cancer murine models, inhibition of mTORC1, which is composed of regulatoryassociated protein of mTOR (RAPTOR), mLST8 (also known as $\mathrm{G} \beta \mathrm{L}$ ), and AKT substrate of $40 \mathrm{kDa}$ (PRAS40), with the mTORi rapamycin, led to MAPK pathway activation through a PI3Kdependent feedback loop. However, when rapamycin was combined with the MEK1/2 inhibitor, PD0325901, MAPK feedback activation was abrogated and resulted in an enhanced antitumoral effect (Carracedo et al, 2008; Kinkade et al, 2008).

We also evaluated the combination of temsirolimus, an inhibitor of mTOR complex 1, and selumetinib (AZD6244, ARRY-142886) for inhibition of STS proliferation in vitro. As detailed below, the combination synergistically inhibited growth of STS cell lines. Thus, cotargeting signalling pathways may be a potential strategy to overcome the mTORi-activated PI3K-Akt feedback pathway, and a clinical trial combining temsirolimus and selumetinib for STS was carried out.

\section{PATIENTS AND METHODS}

Preclinical studies. Human uterine leiomyosarcoma (SK-UT-1), fibrosarcoma (HT1080), and liposarcoma (SW872) cell lines (American Type Culture Collection, Manassas, VA, USA) were treated in triplicate with temsirolimus (1-100 nM) and selumetinib (10-1000 nм) alone or in combination for 6 days. Proliferation was evaluated on a fluorescence-based digital image microscopy system
(DIMSCAN, Bioimaging Solutions Inc., San Diego, CA, USA). The potential for antagonistic, additive, or synergistic interaction of temsirolimus with selumetinib was quantified by the CalcuSyn automated software (Biosoft, Ferguson, MO, USA).

Patients. Adult patients with histologically confirmed STS with metastatic (de novo or recurrent) or locally advanced, unresectable disease were eligible. Patients must have had measurable disease, defined as at least one lesion that could be accurately measured in at least one dimension as $\geqslant 10 \mathrm{~mm}$ with spiral CT scan. Patients may have received $\leqslant 2$ prior chemotherapeutic regimens (single agent or combination chemotherapies), with a life expectancy of at least 12 weeks. Other eligibility criteria included Eastern Cooperative Oncology Group (ECOG) performance status of two or less, absolute neutrophil count $\geqslant 1000 \mathrm{~mm}^{-3}$, platelet count $>100000 \mathrm{~mm}^{-3}$, haemoglobin $>8 \mathrm{~g} \mathrm{dl}^{-1}$, serum creatinine $<1.5 \times$ upper limits of normal (ULN), or calculated creatinine clearance $\geqslant 45 \mathrm{ml} \mathrm{min}^{-1}$, total bilirubin $\leqslant 1.5 \times$ ULN, SGPT $(\mathrm{ALT}) \leqslant 5 \times$ ULN for age, and serum albumin $\geqslant 2 \mathrm{~g} \mathrm{dl}^{-1}$. Patient must have had no evidence of dyspnoea at rest, no exercise intolerance, and a pulse oximetry $>94 \%$ if measured.

Patients were excluded if they had known brain metastases, prior MEK inhibitor use, or received cancer treatments including radiation within three weeks (at least 6 weeks for mitomycin-C and nitrosureas). Patients with pediatric-type sarcomas (Ewing/Ewinglike or rhabdomyosarcoma) were also not eligible. Institutional review board approval was obtained for the study protocol, and all patients provided written informed consent before entering the study.

Study design and assessments. This was a multicentre randomised, open-label, phase 2 study with a goal to accrue 35 patients per arm. (Clinicaltrials.gov identifier: NCT01206140) Randomisation was conducted by the study biostatistician using a permuted block design (block-size of 4), stratified by prior therapy ( 0 vs 1 or 2 ) and sarcoma subtype (liposarcoma, leiomyosarcoma, synovial
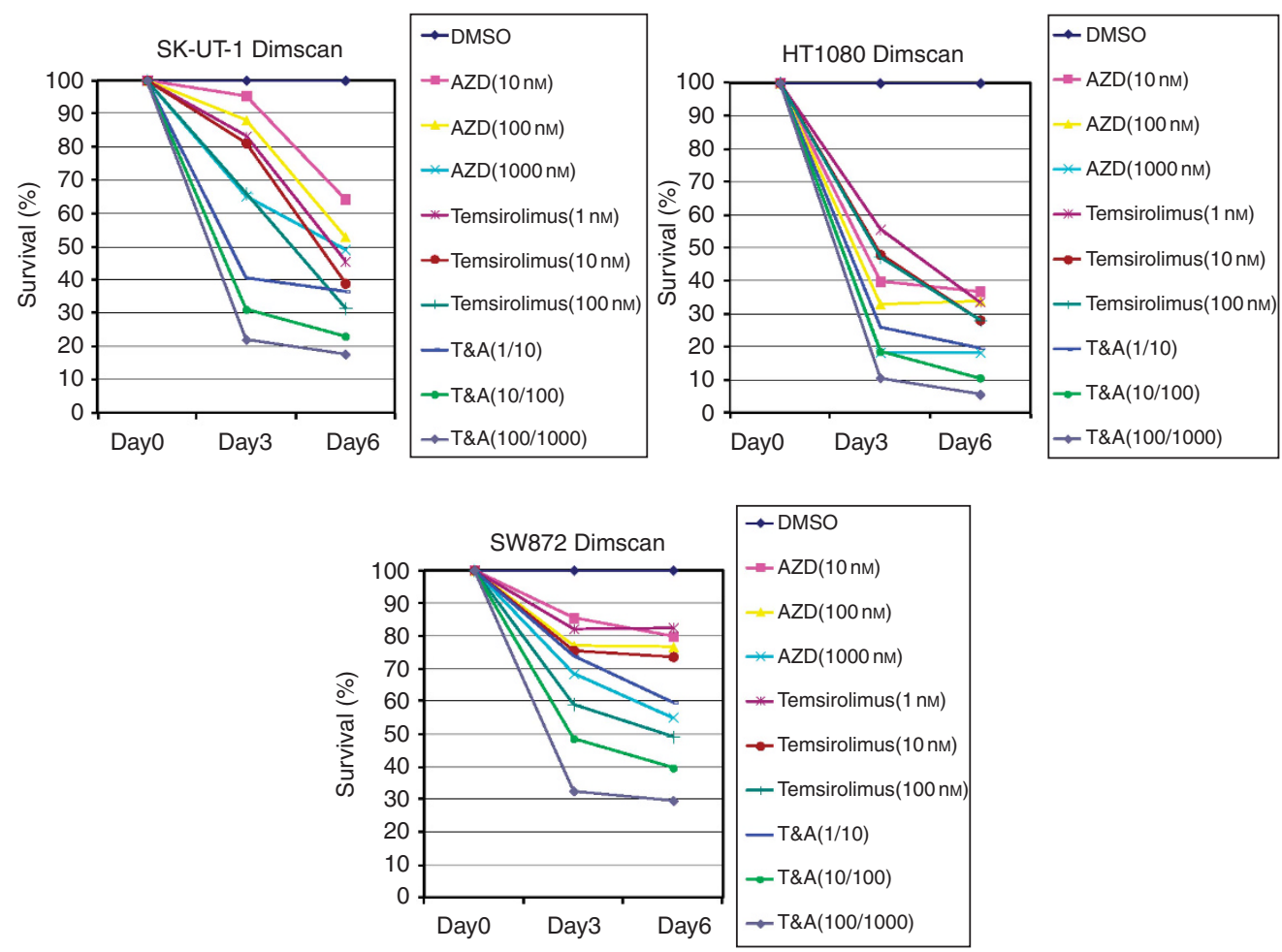

Figure 1. Inhibition of proliferation by temsirolimus, selumetinib (AZD), and the combination (T\&A) in logarithmic concentrations in STS cell lines (SK-UT-1: leiomyosarcoma, HT1080: fibrosarcoma, SW872: liposarcoma) compared with control (dimethyl sulfoxide, DMSO). 
sarcoma, pleomorphic undifferentiated sarcoma, other). Randomisation log was maintained by the central data coordinating centre, and slots were assigned after eligibility was confirmed. The primary endpoint was PFS by RECIST 1.1 criteria. Secondary endpoints were 4-month PFS rate, response rate, and toxicity. Inhibition of activated ERK1/2 in stimulated peripheral blood mononuclear cells (PBMCs), and activation status of mTOR-AKT pathway in tissue biopsies from normal skin were assessed (Ki67, p62, phospho-p70, BCL-2, phospho-AKT, cleaved caspase 3) within 7 days pre- and post-cycle 1 treatment.

The starting dose of selumetinib arm was $75 \mathrm{mg}$ p.o. bid (Arm A), and selumetinib $50 \mathrm{mg}$ bid when combined with temsirolimus at $25 \mathrm{mg}$ i.v. weekly (Arm B). This dosing was based on a phase 1 study where this dosing combination was used (Patel et al, 2013). Disease response was assessed by RECIST 1.1 criteria and Choi criteria (Choi et al, 2007). Patients initially randomized to Arm A were allowed to cross-over to Arm B after progression by RECIST criteria. Each cycle was 4 weeks, with imaging obtained every two cycles until unacceptable toxicity or progressive disease. Adverse events were graded according to National Cancer Institute Common Terminology Criteria of Adverse Events version 4.0.

Correlative studies. Peripheral blood mononuclear cells and normal skin biopsies were obtained pretreatment and after completion of cycle 1. Skin biopsy specimens were bisected in half. One-half were snap-frozen in liquid nitrogen, and the other half were formalin fixed and paraffin embedded. Immunohistochemisty (IHC) for Ki67, p62, phospho-p70, BCL-2, phosphoAKT, cleaved caspase 3 were performed on the formalin fixed and paraffin-embedded specimen, and the phosphorylation state of the signalling proteins was performed in snap-frozen samples.

For PBMC analysis, ERK phosphorylation levels were assessed as a surrogate marker for selumetinib activity. Eight mililiters of peripheral blood was collected in a preload sodium citratecontaining cell preparation tube with 12-O-tetradecanoylphorbol13-acetate pretreatment and after completion of 1 cycle of selumetinib. To activate ERK, the whole blood was treated with 12-O-tetradecanoylphorbol-13-acetate for $10 \mathrm{~min}$ at $37^{\circ} \mathrm{C}$ within $1 \mathrm{~h}$ of being drawn. Peripheral blood mononuclear cells were separated and collected though centrifugation. ERK phosphorylation was preserved by immediate fixation of the cells with $1.2 \%$ methanol-free formaldehyde and transferred to a cryovial tube frozen at $-80^{\circ} \mathrm{C}$. The frozen cell pellets of PBMCs were subsequently thawed and stained with an anti-phospho-ERK antibody (Cell signaling Technology, Inc, Danvers, MA, USA), followed by a fluorescein isothiocyaanate-conjugated secondary antibody detection by fluorescence-activated cell sorting analysis.

Statistical analysis. The accrual goal of 35 patients to each arm would allow a $90 \%$ power at the 0.1 one-sided significance level to detect a hazard ratio (HR) of 2 in favour of the combination arm. Analysis was performed by an as-treated principle. Subgroup analysis was performed on the prespecified sarcoma subgroups, with no adjustment for multiple comparisons in the context of this randomised phase 2 study. Biological correlates were analysed in an exploratory manner.

Outcomes reported on patients who crossed over to the combination arm upon progression were summarised using data before the cross-over from single-agent selumetinib. Progression as per RECIST 1.1 was defined as at least a $20 \%$ increase in the sum of the diameters of target lesions in reference to the smallest sum on study, or the appearance of one or more new lesions, or death. Time to PFS was calculated from date of randomisation to the earliest qualifying event (last date of contact for censored patients with no events), and was summarised by the Kaplan-Meier method. Progression-free suvrvival rates were calculated using the survival distribution function, and $95 \%$ confidence limits were calculated using the log-log transformation. The log-rank test was used to assess differences in PFS by treatment arm overall, as well as by major histology group stratifications. Further, the Cox proportional hazards model was used to estimate hazard ratios between the two treatment arms.

\section{RESULTS}

Preclinical studies. The drug combinations of temsirolimus (1, $10,100 \mathrm{~nm})$ with selumetinib $(10,100,1000 \mathrm{~nm})$, respectively, led to inhibition of proliferation combination Index (CI) values at the lowest concentrations of 0.001 (SK-UT-1, leiomyosarcoma), 0.047 (HT1080, fibrosarcoma), and 0.152 (SW872, liposarcoma). (Figure 1) At the highest concentrations, the corresponding values were $0.024,0.046$, and 0.011 . A CI value $<0.1$ indicates very strong synergism, and $0.1-0.3$ indicates strong synergism as described by Chou and Talalay (Chou and and Talalay, 1984). These results were consistent with strong to very strong synergism in all STS cell lines tested at the lowest concentration of temsirolimus (1 nM) and selumetinib (10 nM). Further, the SK-UT-1 cell line appeared to be the most sensitive cell line by nearly 50-fold over HT1080 and over 150 -fold over SW872 cells. These promising preclinical results led to the current clinical trial.

Patients. A total of 71 patients were enrolled from October 2010 to January 2013 across four sites, City of Hope, University of Pittsburgh, UC Davis, and University of Southern California. Thirty-six patients were randomised to selumetinib alone and 35 patients to the combination arm. (Figure 2B) One patient in the selumetinib arm was found to have bone sarcoma after enrollment and was deemed ineligible owing to incorrect diagnosis, and another patient never started treatment; therefore, 34 selumetinib patients were deemed eligible for analysis. Patient characteristics are reported in Table 1 . Nineteen patients crossed over to the combination after documented progression and received a median of 2 cycles (range 1.7-16.4 months), with 5 patients receiving $\geqslant 6$ cycles after crossover. Patients were followed for a median of 4.5 months in selumetinib arm and 3.4 months with the combination regimen. Study completed full accrual as planned, and follow-up continued until all patients were off therapy.

Clinical outcomes. There was no difference in PFS between the single agent $v s$ combination arm for the overall cohort (median 1.9 vs 2.1 months, $P=0.77$, HR: 0.92). (Figure 3A) However, compared with single agent $(N=10)$, an improved median PFS was observed in the combination arm in the prespecified leiomyosarcoma stratum (median 1.8 vs 3.7 months; $P=0.01$, HR: 4.1). (Figure 3B) For the 19 patients who crossed over to combination arm, 12 of 19 patients eventually progressed; following their cross-over date, median PFS was 5.9 months (95\% CI 1.8-8.5).

Three-month PFS rate was $34.6 \%$ (95\% CI 16-51) with combination and $27.6 \%(13-44)$ for single-agent regimen in the overall cohort; it was $50 \% \quad(95 \%$ CI $0.19-0.81)$ with the combination vs $15 \%(0-51)$ with selumetinib alone in the leiomyosarcoma cohort. Four-month PFS rate was 24\% for both arms in the overall cohort; it was 50\% (95\% CI 0.19-0.81) with the combination $v s 0 \%$ with selumetinib alone in the leiomyosarcoma cohort. In leiomyosarcoma patients, 6-month PFS rate was 37.5\% (11-69) with the combination, vs $0 \%$ with selumetinib alone. (Table 2)

Overall response rate. Two patients with single-agent selumetinib had a partial response per RECIST criteria (confirmed PR in an undifferentiated pleomorphic sarcoma and an unconfirmed PR in a synovial sarcoma patient), and nine patients had stable disease with a median duration of 7.4 months (range: 1.8-20.9). (Figure 3C and D) With the combination regimen, although there were no 


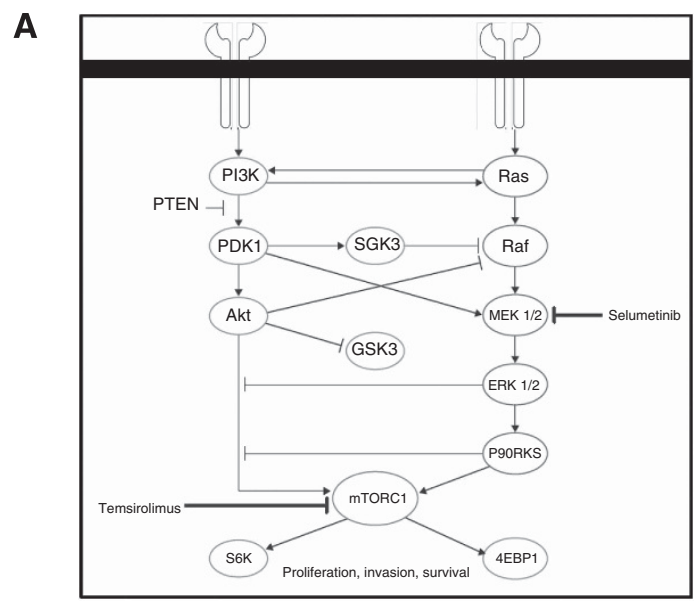

B

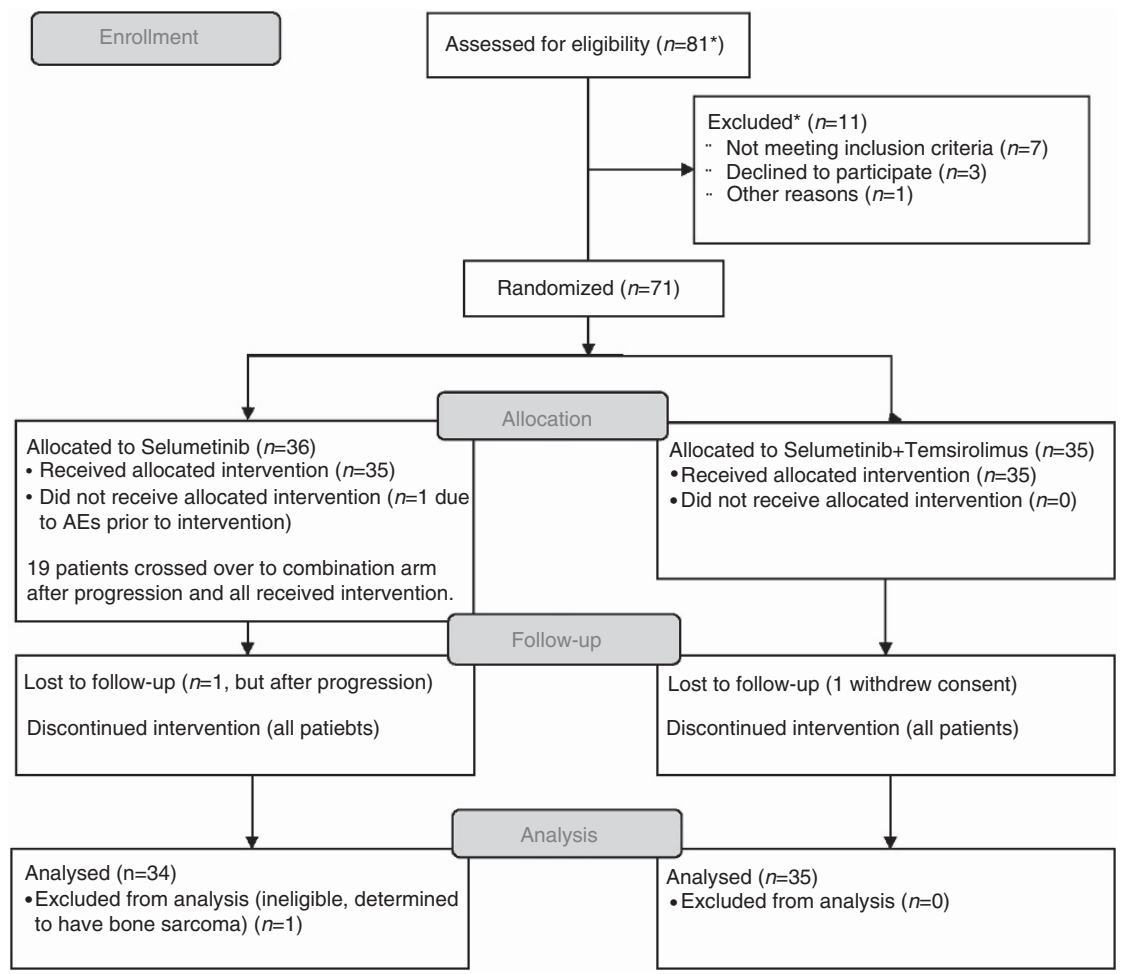

${ }^{*}$ Consent forms and screening at all sites were not tracked until accrual was close to completion. The number assessed for eligibility is an estimate.

Figure 2. (A) Ras-PI3K-mTORC1 pathway; (B) CONSORT diagram.

objective responses seen per RECIST, 12 patients had stable disease, with a median duration of 2.1 months (range: 1.7-22.2). Five $v s$ two patients in the leiomyosarcoma cohort had stable disease with the combination arm $v s$ selumetinib alone, with no objective responses seen in either arm. Nineteen of 34 selumetinib patients crossed over to the combination; 2 patients had a partial response per RECIST, whereas 6 (32\%) patients had stable disease, and 11 (58\%) had progressive disease. With Choi response criteria analysis, five patients with selumetinib alone and four in the combination arm had PR; there were two PRs in the combination arm of the leiomysarcoma cohort and zero with selumetinib alone.

Toxicity and dose modifications. On the basis of an early interim review of toxicities, grade 3 mucositis/stomatitis was noted in four of 11 patients randomised to the combination arm or crossed over from single-agent selumetinib. Therefore, the study was amended to reduce the temsirolimus dose from $25 \mathrm{mg}$ to $20 \mathrm{mg}$ i.v. weekly. Of the 35 patients randomised to the combination regimen, 16 patients received the $25-\mathrm{mg}$ dose, whereas 19 received $20 \mathrm{mg}$. Table 3 shows grade 3 and 4 adverse events in all patients before cross-over.

Oral mucositis was the most frequent grade 3 adverse event; it was observed in $29 \%$ of all patients in the combination arm. Lymphopenia (26\%), neutropenia, and anaemia (20\% each) were the next most common grade 3 events in this arm. For the selumetinib alone arm, hypertension (12\%) was the most common grade 3 event. Most frequent grade 1/2 toxicities in the selumetinib alone and combination arms included acneiform rash (59\% vs 43\%, respectively), fatigue ( $53 \%$ vs $43 \%)$, anaemia ( $24 \%$ vs $48 \%$ ) diarrhoea (37\% vs $47 \%$ ), and nausea ( $47 \%$ vs $31 \%$ ).

All patients are off study, with the majority owing to progression of disease $(61.8 \%$ in the single-agent and $74.3 \%$ in 
Table 1. Patient characteristics

\begin{tabular}{|c|c|c|}
\hline Patient characteristics & $\begin{array}{l}\text { Selumetinib alone } \\
\qquad(n=34)\end{array}$ & $\begin{array}{l}\text { Selumetinib + temsirolimus } \\
\qquad(n=35)\end{array}$ \\
\hline $\begin{array}{l}\text { Age at randomisation, median (range) } \\
\text { Gender, } N(\%) \text { male } \\
\text { Race/ethnicity, } N(\%) \text { Caucasian }\end{array}$ & $\begin{array}{l}56.2(34.4-84.3) \\
17(50.0 \%) \\
17(50.0 \%)\end{array}$ & $\begin{array}{l}56.9(20.2-83.7) \\
10(28.6 \%) \\
24(68.6 \%)\end{array}$ \\
\hline \multicolumn{3}{|l|}{ Performance status, $N(\%)$} \\
\hline $\begin{array}{l}0 \\
1 \\
2\end{array}$ & $\begin{array}{r}8(23.5 \%) \\
21(61.8 \%) \\
5(14.7 \%)\end{array}$ & $\begin{array}{l}14(40.0 \%) \\
19(54.3 \%) \\
2(5.7 \%)\end{array}$ \\
\hline \multicolumn{3}{|l|}{ Tumor grade, $\mathbf{N}(\%)$} \\
\hline $\begin{array}{l}\text { Low grade } \\
\text { Intermediate grade } \\
\text { High grade } \\
\text { Unknown }\end{array}$ & $\begin{array}{c}5 \text { (14.7\%) } \\
3 \text { ( }(8.8 \%) \\
24(70.6 \%) \\
2 \text { (5.9\%) }\end{array}$ & $\begin{aligned} 5 & (14.3 \%) \\
2 & (5.7 \%) \\
24 & (68.6 \%) \\
4 & (11.4 \%)\end{aligned}$ \\
\hline \multicolumn{3}{|l|}{ Invasion depth, $N(\%)$} \\
\hline $\begin{array}{l}\text { Superficial } \\
\text { Deep } \\
\text { Unknown }\end{array}$ & $\begin{array}{r}4(11.8 \%) \\
26(76.5 \%) \\
4(11.8 \%)\end{array}$ & $\begin{array}{r}5(14.3 \%) \\
23(65.7 \%) \\
7(20.0 \%)\end{array}$ \\
\hline \multicolumn{3}{|l|}{ Stratum, $N(\%)$} \\
\hline $\begin{array}{l}\text { Liposarcoma, prior therapy } 0 \\
\text { Liposarcoma, prior therapy } 1 \text { or } 2 \\
\text { Pleomorphic undifferentiated sarcoma, prior therapy } 1 \text { or } 2 \\
\text { Synovial sarcoma, prior therapy } 1 \text { or } 2 \\
\text { Leiomyosarcoma, prior therapy } 1 \text { or } 2 \\
\text { Other, prior therapy } 0 \\
\text { Other, prior therapy } 1 \text { or } 2\end{array}$ & $\begin{aligned} & 3(8.8 \%) \\
& 2(5.9 \%) \\
& 2(5.9 \%) \\
& 2(5.9 \%) \\
& 10(29.4 \%) \\
& 2(5.9 \%) \\
& 13(38.2 \%) \\
&\end{aligned}$ & $\begin{aligned} & 2(5.7 \%) \\
& 2(5.7 \%) \\
& 2(5.7 \%) \\
& 3(8.6 \%) \\
& 11(31.4 \%) \\
& 2(5.7 \%) \\
& 13(37.1 \%) \\
&\end{aligned}$ \\
\hline \multicolumn{3}{|l|}{ Prior therapy, $N(\%)$} \\
\hline $\begin{array}{l}\text { No prior chemotherapy } \\
\text { Noncytotoxic drugs only }\end{array}$ & $\begin{array}{l}7(20.6 \%) \\
1(2.9 \%) \\
\end{array}$ & $\begin{array}{l}2(5.7 \%) \\
2(5.7 \%) \\
\end{array}$ \\
\hline \multicolumn{3}{|l|}{ Cytotoxic drugs } \\
\hline $\begin{array}{l}\text { Anthracycline based only } \\
\text { Nonanthracycline based only } \\
\text { Both }\end{array}$ & $\begin{array}{r}5(14.7 \%) \\
8(23.5 \%) \\
13(38.2 \%)\end{array}$ & $\begin{aligned} 1 & (2.9 \%) \\
7 & (20.0 \%) \\
23 & (65.7 \%)\end{aligned}$ \\
\hline $\begin{array}{l}\text { Number of prior lines of therapy, median } \\
\text { Total cycles precross-over, median (range) } \\
\text { Total cycles including postcross-over, median (range) }\end{array}$ & $\begin{array}{c}1 \\
2(1-24) \\
4(1-24) \\
\end{array}$ & $\begin{array}{c}1 \\
2(1-24) \\
2(1-24) \\
\end{array}$ \\
\hline
\end{tabular}

the combination arm.) Four patients withdrew consent, and 11 patients were removed from the study owing to toxicity (eight in selumetinib alone and three in combination arm.)

Correlative studies. Twenty-eight patients had PBMC samples analysed both before and at end of cycle 1 . In every patient except for three in selumetinib alone and two in combination arm, decrease in ERK phosphorylation was observed posttreatment. (Figure 4) Thus, selumetinib inhibited ERK phosphorylation at both the 50 and $75 \mathrm{mg}$ twice daily schedule. Pre- and posttreatment skin biopsies were obtained simultaneously with the PBMCs to evaluate as a surrogate for tumor mTOR-Akt pathway pharmacodynamic markers including: phospho-p70, phosphoAKT, Ki67 (proliferation), p62 (autophagic flux), BCL-2 (antiapoptosis), and cleaved caspase 3 (apoptosis). However, IHC demonstrated marked variable detection of these proteins in both the pre- and post-treatment specimens. The antibodies used did not reliably detect the desired antigen in the pretreatment skin biopsies. Further, in several samples there was inadequate dermis within the punch skin biopsy specimens for review. Accordingly, IHC of skin biopsies as a surrogate marker for tumour tissue was uninformative for the proteins evaluated in this study.

\section{DISCUSSION}

There was no difference in PFS observed between selumetinib alone and selumetinib with temsirolimus in 69 advanced STS patients who had up to two lines of prior therapy in this multicentre randomised phase 2 clinical trial. The MEK inhibitors have not been previously tested in STS to our knowledge, but do not appear to show significant activity in this patient population. Mammalian target of rapamycin inhibitors have been tested in other trials of advanced STS with varying results. In a first-line phase 2 study of $25 \mathrm{mg}$ weekly temsirolimus in 41 advanced STS patients, median time to progression was only 2 months, with 6 -month PFS rate of $13 \%$ seen (Okuno et al, 2011). However, in a phase 2 trial of another mTORi, ridaforolimus, in mostly pretreated STS patients, a median PFS 15.3 weeks, and 6-month PFS rate of $23.4 \%$ was observed (Chawla et al, 2012).

Whereas the initial dose of temsirolimus in this trial was $25 \mathrm{mg}$ i.v. weekly, owing to the frequency of grade 3 mucositis observed, the starting dose was reduced to $20 \mathrm{mg}$ i.v. weekly. Thus, $46 \%$ of patients received 20-mg dosing of temsirolimus in the combination arm. The FDA approved dosing of temsirolimus for advanced renal cell cancer is $25 \mathrm{mg}$ i.v. weekly; however, it is not clear whether the amended lower dose of temsirolimus in this study can inhibit mTOR signalling as completely. There was a lower incidence of grade 3 mucositis after the dose was lowered; the side effects of the combination appeared to be tolerable at this dosing.

There were no significant difference in response rates between the two arms regardless of response criteria used. Choi criteria were originally developed and validated in assessing GIST response. It has been suggested that Choi criteria may be more accurate in predicting outcomes in STS than RECIST (Stacchiotti et al, 2012), although its use is not currently validated in STS. 
A

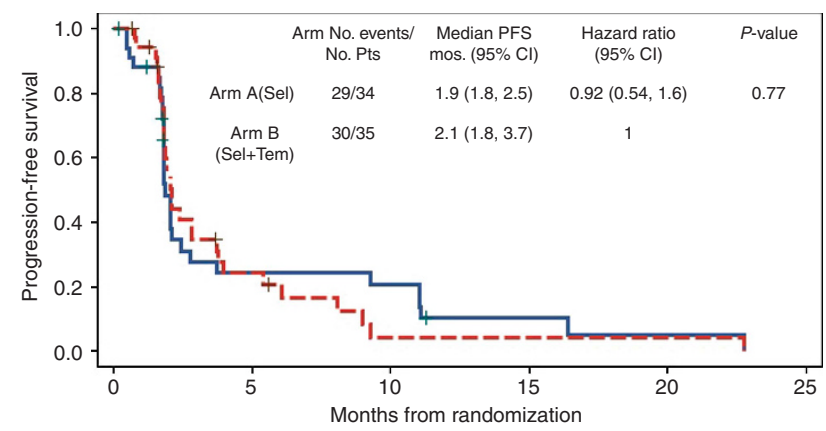

At risk:

ARM B 35

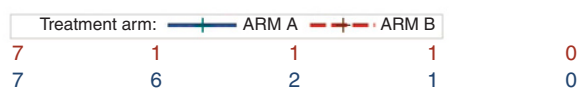

B

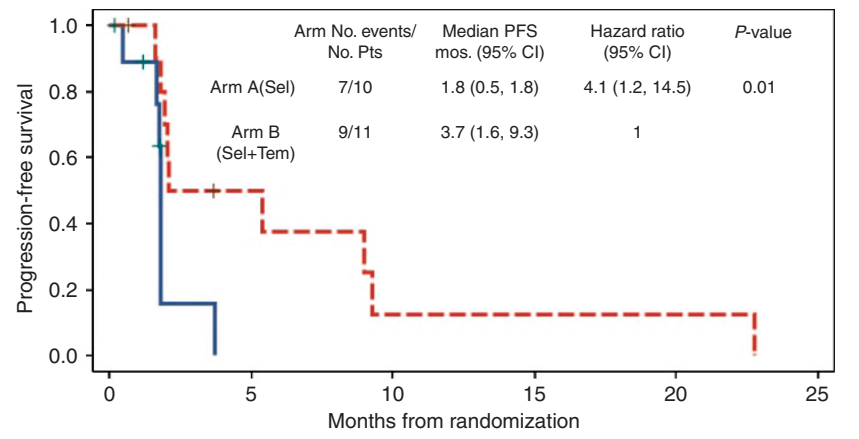

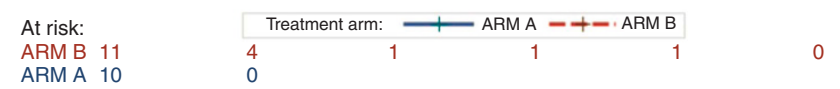

C

Best percent change in sum of target lesion dimensions
(from baseline) All patients

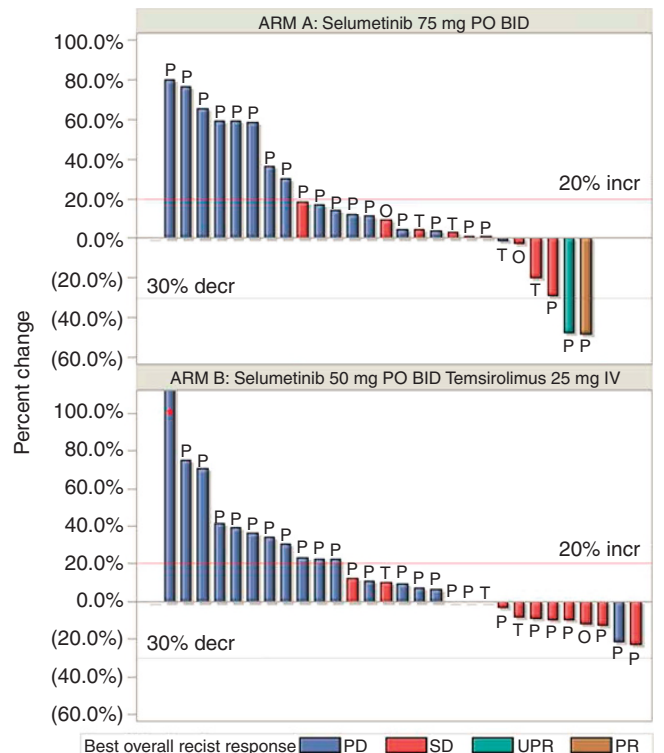

Best overall recist response $\square$ PD $\square$ SD $\square$ UPR $\square$ PR

$P$ : off-tx due to progression; $T$ : off-tx due to toxicity; $O$ : off tx other reason *Patient had $220 \%$ increase

Patients lacking baseline or sufficient follow-up measurements could not be included. Measurements for cross-over patients on Arm A were only used if they were assessed prior to cross-over.
D

Best percent change in sum of targe
(from baseline)

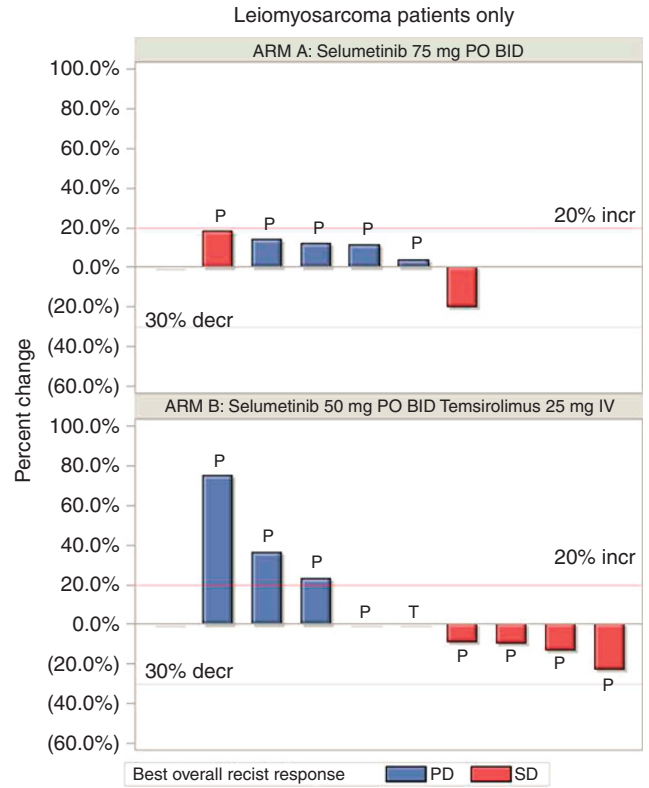

$P$ : off-tx due to progression; $T$ : off-tx due to toxicity; $\mathrm{O}$ : off tx other reason

Figure 3. Progression-free survival (A) All patients (B) leiomyosarcoma cohort waterfall plots of response (by RECIST) (C) All patients (D) Leiomyosarcoma cohort.

A larger number of partial responses were observed with the Choi vs RECIST criteria (9 vs 2).

There was an improvement in PFS and PFS rates observed with the combination arm in the 21-patient leiomyosarcoma cohort; all patients in this cohort had 1 or 2 prior treatments. In a European
Organisation for Research and Treatment of Cancer analysis of phase 2 trials for advanced sarcomas, the 3- and 6- month PFS rates for pretreated STS patients receiving an active agent were 39\% and $14 \%$, respectively. (Van Glabbeke et al, 2002) For second-line therapy, a 3 -month PFS of $\geqslant 40 \%$ was suggested as a benchmark 


\begin{tabular}{|c|c|c|c|c|c|}
\hline Selumetinib arm & PFS rate & $95 \% \mathrm{Cl}$ & Sel + Tem arm & PFS rate & $95 \% \mathrm{Cl}$ \\
\hline \multicolumn{6}{|l|}{ All patients } \\
\hline 3 months & $27.60 \%$ & $13-44$ & 3 months & $34.60 \%$ & $16-51$ \\
\hline 4 months & $24.00 \%$ & $9-40$ & 4 months & $24.00 \%$ & $9-39$ \\
\hline 6 months & $24.20 \%$ & $11-14$ & 6 months & $20.80 \%$ & $9-36$ \\
\hline \multicolumn{6}{|c|}{ Leiomyosarcoma cohort } \\
\hline 3 months & $15.00 \%$ & $0-51$ & 3 months & $50.00 \%$ & $19-81$ \\
\hline 4 months & $0.00 \%$ & 0 & 4 months & $50.00 \%$ & $19-81$ \\
\hline 6 months & $0.00 \%$ & 0 & 6 months & $37.50 \%$ & $11-69$ \\
\hline
\end{tabular}

Table 3. Grade $3 / 4$ adverse events

\begin{tabular}{|c|c|c|c|c|}
\hline \multirow[b]{3}{*}{ Grade 3 adverse events in $>1$ patient and grade 4 events } & \multicolumn{4}{|c|}{ Treatment arm } \\
\hline & \multicolumn{2}{|c|}{ Selumetinib $(n=34)$} & \multicolumn{2}{|c|}{ Sel $+\operatorname{Tem}(n=35)^{a}$} \\
\hline & Grade 3 & Grade 4 & Grade 3 & Grade 4 \\
\hline Anaemia & $2(6 \%)$ & & $7(20 \%)$ & \\
\hline Mucositis oral & & & 10(29\%) & \\
\hline Nausea & $2(6 \%)$ & & $2(6 \%)$ & \\
\hline Vomiting & $1(3 \%)$ & & $3(9 \%)$ & \\
\hline Fatigue & $2(6 \%)$ & & $1(3 \%)$ & \\
\hline Lymphopenia & $1(3 \%)$ & $1(3 \%)$ & $9(26 \%)$ & \\
\hline Neutropenia & $1(3 \%)$ & & $7(20 \%)$ & \\
\hline Thrombocytopenia & & & $2(6 \%)$ & \\
\hline Leukopenia & $1(3 \%)$ & & $3(9 \%)$ & \\
\hline Dehydration & $1(3 \%)$ & & $2(6 \%)$ & \\
\hline Hypokalemia & $2(6 \%)$ & $1(3 \%)$ & & \\
\hline Syncope & $2(6 \%)$ & & $1(3 \%)$ & \\
\hline Rash acneiform & $1(3 \%)$ & & $2(6 \%)$ & \\
\hline Hypertension & $4(12 \%)$ & & $1(3 \%)$ & \\
\hline Thromboembolic event & & & & $1(3 \%)$ \\
\hline
\end{tabular}

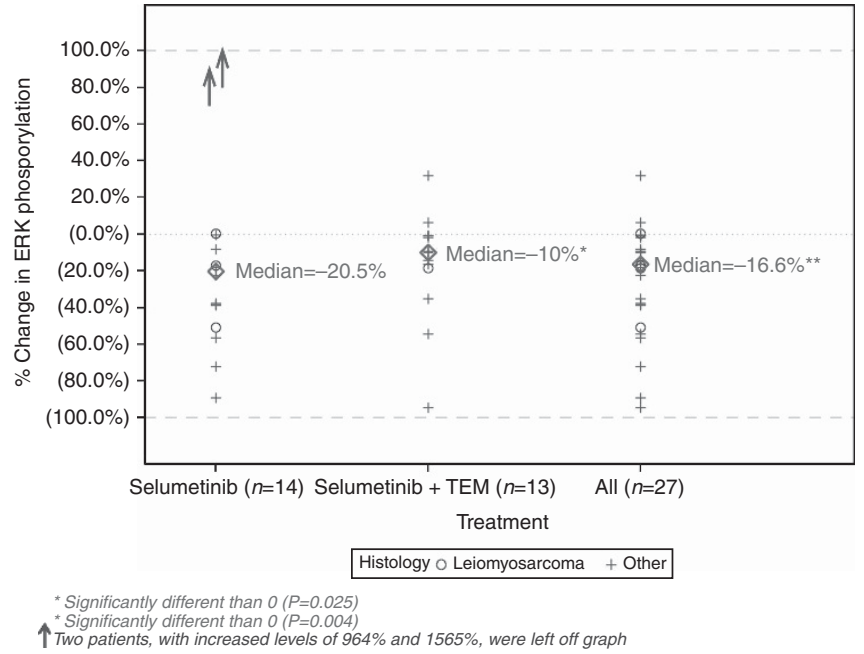

Figure 4. Change in ERK phosphorylation in PBMCs pre to posttreatment.

for drug activity. In the leiomyosarcoma cohort of the current study, a 3-month PFS of 50\% (95\% CI 19-81) was observed, which suggests clinically meaningful activity in this group. In the European Organisation for Research and Treatment of Cancer phase 2 study of pazopanib, the 3-month PFS rate was $44 \%$ for the 41 patients in the leiomyosarcoma cohort, for which $76 \%$ received prior chemotherapy for advanced disease. (Sleijfer et al, 2009)
Although our results compare favourably with these outcomes, this is only based on a 21-patient subgroup analysis.

In leiomyosarcomas, the Akt/mTOR pathway has been shown to be activated and associated with worse prognosis (Setsu et al, 2012). Setsu et al (2012) reported over $70 \%$ of primary leiomyosarcoma tumours demonstrated phosphorylated forms of Akt, mTOR, ribosomal protein S6 kinase (S6), and eukaryotic translation initiation factor $4 \mathrm{E}-$ binding protein (4E-BP1). Interestingly, PTEN expression was lost in only $20 \%$ of the tumours, and mutational analysis failed to reveal any PIK3CA or AKT1 mutations. Also consistent with those findings, absence of genetic alterations in the AKT1, PI3K, PTEN, and EGFR genes in leiomyosarcoma stem-like cells was reported, whereas proteins downstream of the PI3/AKT and MAPK/ERK pathways were strongly activated (Sette et al, 2012). The PTEN gene is located on chromosome $10 \mathrm{q}$, and partial loss of $10 \mathrm{q}$ is also a frequent event in leiomyosarcomas. (Hu et al, 2005; Meza-Zepeda et al, 2006) Mice with smooth muscle lineage-specific knockout of PTEN also develop widespread smooth muscle cell hyperplasia and abdominal leiomyosarcomas, significantly implicating PTEN downregulation in leiomyosarcomagenesis. (Hernando et al, 2007) KRAS mutations can also activate the Akt/mTOR pathway, and KRAS mutations have been reported in a subset (7-33\%) of leiomyosarcoma patients (Hill et al, 1997; Yoo and Robinson, 1999); MEK inhibitors act downstream of RAS in the MAPK pathway. These results may explain the relative sensitivity of leiomyosarcoma to the combination of temsirolimus and selumetinib as compared with selumetinib alone in this study.

Our results are also consistent with a clinical report evaluating temsirolimus in a small cohort of subjects with advanced 
leiomyosarcomas. (Italiano et al, 2011) Although no objective responses were observed by RECIST criteria, stable disease was achieved in three of six subjects. These three subjects had partial response according to the Choi criteria. Given the lack of a temsirolimus alone comparator arm in this study, it is difficult to determine what benefit is gained by adding selumetinib to temsirolimus, and it is possible a similar outcome may have been observed with single-agent temsirolimus in leiomyosarcoma patients in our study as well.

In summary, although no improvement in PFS was seen in advanced STS patients with selumetinib alone or with addition of temsirolimus, the combination may have clinically meaningful activity in leiomyosarcoma patients, with improved median PFS, and an acceptable side effect profile. On the basis of these findings, we feel that testing of the combination regimen $v s$ single-agent temsirolimus would be warranted in a randomised, phase 2 trial in leiomyosarcoma patients.

\section{ACKNOWLEDGEMENTS}

This work was supported by the National Cancer Institute at the National Institutes of Health: NO1-CM-2011-00038, and National Comprehensive Cancer Network Temsirolimus Oncology Research Program. Selumetinib was provided by AstraZeneca. Temsirolimus was provided by Pfizer.

\section{CONFLICT OF INTEREST}

The authors declare no conflict of interest.

\section{REFERENCES}

Carracedo A, Ma L, Teruya-Feldstein J, Rojo F, Salmena L, Alimonti A, Egia A, Sasaki AT, Thomas G, Kozma SC, Papa A, Nardella C, Cantley LC, Baselga J, Pandolfi PP (2008) Inhibition of mTORC1 leads to MAPK pathway activation through a PI3K-dependent feedback loop in human cancer. J Clin Investig 118(9): 3065-3074.

Chawla SP, Staddon AP, Baker LH, Schuetze SM, Tolcher AW, D'Amato GZ, Blay JY, Mita MM, Sankhala KK, Berk L, Rivera VM, Clackson T, Loewy JW, Haluska FG, Demetri GD (2012) Phase II study of the mammalian target of rapamycin inhibitor ridaforolimus in patients with advanced bone and soft tissue sarcomas. J clin oncol 30(1): 78-84.

Choi H, Charnsangavej C, Faria SC, Macapinlac HA, Burgess MA, Patel SR, Chen LL, Podoloff DA, Benjamin RS (2007) Correlation of CT and PET in patients with metastatic GIST treated at a single institution with imatinib mesylate: proposal of new CT response criteria. J Clin Oncol 25: 1753-1759.

Chou TC, Talalay P (1984) Quantitative analysis of dose-effect relationships: the combined effects of multiple drugs or enzyme inhibitors. Adv Enzyme Regul 22: 27-55.

Hernando E, Charytonowicz E, Dudas ME, Menendez S, Matushansky I, Mills J, Socci ND, Behrendt N, Ma L, Maki RG, Pandolfi PP, Cordon-Cardo C (2007) The AKT-mTOR pathway plays a critical role in the development of leiomyosarcomas. Nat Med 13(6): 748-753.

Hill MA, Gong C, Casey TJ, Menon AG, Mera R, Gillespie AT, Giardina JF, Levine EA, Hunt JD (1997) Detection of K-ras mutations in resected primary leiomyosarcoma. Cancer Epidemiol Biomarkers Prev 6(12): 1095-1100.

Hu J, Rao UN, Jasani S, Khanna V, Yaw K, Surti U (2005) Loss of DNA copy number of $10 \mathrm{q}$ is associated with aggressive behavior of leiomyosarcomas: a comparative genomic hybridization study. Cancer Genet Cytogenet 161(1): 20-27.
Italiano A, Kind M, Stoeckle E, Jones N, Coindre JM, Bui B (2011) Temsirolimus in advanced leiomyosarcomas: patterns of response and correlation with the activation of the mammalian target of rapamycin pathway. Anticancer Drugs 22(5): 463-467.

Kinkade CW, Castillo-Martin M, Puzio-Kuter A, Yan J, Foster TH, Gao H, Sun Y, Ouyang X, Gerald WL, Cordon-Cardo C, Abate-Shen C (2008) Targeting AKT/mTOR and ERK MAPK signaling inhibits hormonerefractory prostate cancer in a preclinical mouse model. J Clin Investig 118(9): 3051-3064.

Meza-Zepeda LA, Kresse SH, Barragan-Polania AH, Bjerkehagen B, Ohnstad HO, Namlos HM, Wang J, Kristiansen BE, Myklebost O (2006) Array comparative genomic hybridization reveals distinct DNA copy number differences between gastrointestinal stromal tumors and leiomyosarcomas. Cancer Res 66(18): 8984-8993.

Okuno S, Bailey H, Mahoney MR, Adkins D, Maples W, Fitch T, Ettinger D, Erlichman C, Sarkaria JN (2011) A phase 2 study of temsirolimus (CCI-779) in patients with soft tissue sarcomas: a study of the Mayo phase 2 consortium (P2C). Cancer 117(15): 3468-3475.

Patel SP, Lazar AJ, Papadopoulos NE, Liu P, Infante JR, Glass MR, Vaughn CS, LoRusso PM, Cohen RB, Davies MA, Kim KB (2013) Clinical responses to selumetinib (AZD6244; ARRY-142886)-based combination therapy stratified by gene mutations in patients with metastatic melanoma. Cancer 119(4): 799-805.

Sabatini DM (2006) mTOR and cancer: insights into a complex relationship. Nat Rev Cancer 6(9): 729-734.

Setsu N, Yamamoto H, Kohashi K, Endo M, Matsuda S, Yokoyama R, Nishiyama K, Iwamoto Y, Dobashi Y, Oda Y (2012) The Akt/mammalian target of rapamycin pathway is activated and associated with adverse prognosis in soft tissue leiomyosarcomas. Cancer 118(6): 1637-1648.

Sette G, Salvati V, Memeo L, Fecchi K, Colarossi C, Di Matteo P, Signore M, Biffoni M, D’Andrea V, De Antoni E, Canzonieri V, De Maria R, Eramo A (2012) EGFR inhibition abrogates leiomyosarcoma cell chemoresistance through inactivation of survival pathways and impairment of CSC potential. PLoS One 7(10): e46891.

Sleijfer S, Ray-Coquard I, Papai Z, Le Cesne A, Scurr M, Schoffski P, Collin F, Pandite L, Marreaud S, De Brauwer A, van Glabbeke M, Verweij J, Blay JY (2009) Pazopanib, a multikinase angiogenesis inhibitor, in patients with relapsed or refractory advanced soft tissue sarcoma: a phase II study from the European organisation for research and treatment of cancer-soft tissue and bone sarcoma group (EORTC study 62043). J Clin Oncol 27(19): 3126-3132.

Stacchiotti S, Verderio P, Messina A, Morosi C, Collini P, Llombart-Bosch A, Martin J, Comandone A, Cruz J, Ferraro A, Grignani G, Pizzamiglio S, Quagliuolo V, Picci P, Frustaci S, Dei Tos AP, Casali PG, Gronchi A (2012) Tumor response assessment by modified Choi criteria in localized high-risk soft tissue sarcoma treated with chemotherapy. Cancer 118(23): 5857-5866.

van der Graaf WT, Blay JY, Chawla SP, Kim DW, Bui-Nguyen B, Casali PG, Schoffski P, Aglietta M, Staddon AP, Beppu Y, Le Cesne A, Gelderblom H, Judson IR, Araki N, Ouali M, Marreaud S, Hodge R, Dewji MR, Coens C, Demetri GD, Fletcher CD, Dei Tos AP, Hohenberger P (2012) Pazopanib for metastatic soft-tissue sarcoma (PALETTE): a randomised, double-blind, placebo-controlled phase 3 trial. Lancet 379(9829): 1879-1886.

Van Glabbeke M, Verweij J, Judson I, Nielsen OS (2002) Progression-free rate as the principal end-point for phase II trials in soft-tissue sarcomas. Eur J Cancer 38(4): 543-549.

Wan X, Helman LJ (2007) The biology behind mTOR Inhibition in sarcoma. Oncologist 12(8): 1007-1018.

Yoo J, Robinson RA (1999) H-ras and K-ras mutations in soft tissue sarcoma: comparative studies of sarcomas from Korean and American patients. Cancer 86(1): 58-63.

(c) (1) (2) This work is licensed under the Creative Commons Attribution-Non-Commercial-Share Alike 4.0 International License. To view a copy of this license, visit http:// creativecommons.org/licenses/by-nc-sa/4.0/ 